\title{
Pedestrian Evacuation with Asymmetrical Exit Based on an Improved Cellular Automata
}

\author{
Nuo Zhu* \\ Information center of CAAC, 155\# Dongsi Xidajie, Beijing, 100710, China \\ ${ }^{*}$ Corresponding author
}

\begin{abstract}
Simulation of pedestrian evacuation flow from a room with asymmetrical exit layout is presented based on cellular automata (CA) in this paper. Pedestrian moving rules were established, according to two-dimensional cellular automaton Moore neighborhood. The dynamic parameters: Directionparameter, Empty-parameter and Cognition-parameter are formulated to simplify tactically the decision-making process of pedestrians, considering the effects of pedestrian jam around exits and the width of exits on evacuation path selection in order to reduce evacuation imbalance caused by the asymmetry of exits layout, which can reflect the pedestrian judgment on the surrounding conditions and decide the pedestrian's choice of action. The simulation results of the improved and previous models are compared and analyzed. Simulation results show that evacuation time $\boldsymbol{T}$ increases nonlinearly (exponential distribution) when system size is increased, there is a linear relationship between evacuation time and pedestrian density, however, there is a negative exponential relationship between evacuation time and exit width. At last, the best exit layout is given in this paper. The simulation results correspond with the actual, it is instructional significant for pedestrian evacuation.
\end{abstract}

Keywords-cellular automata; pedestrian evacuation; evacuation time; asymmetrical exit layout.

\section{INTRODUCTION}

Recent research has shown that complex behavior phenomena in pedestrian flows and pedestrian evacuation can be successfully studied from a physical viewpoint ([1-8]). Based on the characteristics of individual pedestrian and system surroundings, these simulation models are extended, combined or modified to approximate pedestrian dynamics. A new parameter right-hand parameter is introduced in the Dynamic Parameters Model [9] to generalize pedestrians' walking preference. In most pedestrian evacuation simulations, the room is supposed to be a rectangle box with one exit. However, rooms with more than one exit can be frequently observed in realistic life, and the exits layout will affect the pedestrian evacuation process. Pedestrians' exit selection strategy will be a basic rule in the evacuation simulation. To the best of our knowledge, apart from the logit-based discrete choice being used to formulate the exit choice behavior and the pedestrian density around exits being considered in the pedestrian movement rules, there is relatively few evacuation research, particularly in the asymmetrical layout of multi-exits. In some models [9-12], if more than one possible target locations, one of them will be chosen as the target position randomly with equal probability. At the same time, when a conflict occurs when any two or more pedestrians attempt to move to the same target position, one of them will be also chosen randomly with equal probability. In fact, according to the location of the pedestrian and the distance between target location (or exits) and pedestrian, the probability is different. But many models [9-12] have been dealt it with the same probability. We believe that this is not realistic. It is well accepted that rational pedestrians are intelligent and adaptive to the dynamic conditions around by constantly seeking and choosing an optimum route. So pedestrians, who are familiar with evacuation circumstances without panics and intend to leave room within the shortest possible time, will try to reduce the evacuation imbalance caused by the asymmetry of exits layout. In the previous models [10-12], crowd distribution is not considered or it is supposed that the crowd is uniformly distributed in a large room without any obstacle, but this parameter is important in specifying doors locations. In this paper, the Dynamic Parameter Model is presented to simulate pedestrian multi-exit evacuation, which can also be adapted to the simulation with one exit. The dynamic parameters are formulated to instruct pedestrians' exit selection strategy. In the following sections, Section II and III describe the pedestrian evacuation model and the simulation procedures. The simulation results are presented in Section IV. The conclusion is given in Section V.

\section{MODEL DESCRIPTION}
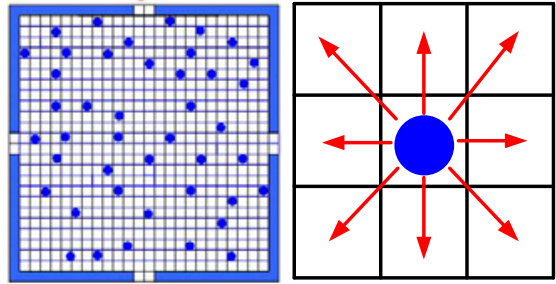

\begin{tabular}{|l|l|l|}
\hline$P_{-1,-1}$ & $\mathbf{P}_{-1,0}$ & $\mathbf{P}_{-1,1}$ \\
\hline $\mathbf{P}_{0,-1}$ & $\mathbf{P}_{0,0}$ & $\mathbf{P}_{0,1}$ \\
\hline $\mathbf{P}_{1,-1}$ & $\mathbf{P}_{1,0}$ & $\mathbf{P}_{1,1}$ \\
\hline
\end{tabular}

(a) simulation system

(b) movement field

(c) matrix of transition payoffs

\section{FIGURE I. SIMULATION MODEL OF EVACUATION PEDESTRIAN}

The model proposed on $(\mathrm{W}+2) \times(\mathrm{W}+2)$ twodimensional discrete grid, where the movement area in the system is marked out into $\mathrm{W} \times \mathrm{W}$ cells and $\mathrm{W}$ is the system size. Discrete cellular has the same size, barriers occupy the boundary's cellular and consist of the wall, some empty cellular left on the wall as an exit. In each discrete time step, pedestrian can choose to wait or move to the next possible position (see Figure I(b)). The transition payoff (a $3 \times 3$ matrix) is introduced to describe the relative advantages to be obtained 
by a certain pedestrian (see Figure I(c)) through Directionparameter, Empty-parameter and Cognition-parameter.Once the geometry of the system is determined, each cell is assigned a constant value representing its distance to the door.

Direction-parameter indicates the cell's degree of approximation to the pedestrian destination, namely room exit with a single step in a time step. The values of elements of Direction-parameter matrix are given as:

$$
D_{i j}=\frac{S_{00}-S_{i j}}{\sqrt{i^{2}+j^{2}}}
$$

Where $S_{00}$-the shortest distance from the core cell in the movement field to the evacuation exits. $S_{i j}$-the shortest distance from the cell (i, j) in the movement field to the evacuation exits.

Empty-parameter indicates whether the cell is occupied or not. The values of elements of Empty-parameter matrix:

$$
E_{i j}=\left\{\begin{array}{lr}
\max \left(D_{i j}\right), & \text { empty cells, } \\
0, & \text { the core cell, } \\
-\max \left(D_{i j}\right), & \text { occupy cells }
\end{array}\right.
$$

Cognition-parameter describes the effects of pedestrian jam around exits and the width of exits. The values of elements of Cognition-parameter matrix are given as:

$$
C_{i j}=\max \left(\max _{m}\left(\frac{d_{m} \cdot\left(p_{\text {in }}^{t} / S_{i n}^{t}\right)}{d_{L} \cdot\left(p_{m}^{t} / S_{m}^{\mathrm{t}}\right)}\right)\right)
$$

Where $d_{m}$-the width of m-th exits; $d_{L}$-the sum width of exits; $p_{\text {in }}^{t}$-the number of pedestrians in the evacuation system at $\mathrm{t}$ time step; $p_{\mathrm{m}}^{t}$-the number of pedestrians around $\mathrm{m}$-th exits at time step; ${ }^{\mathrm{t}}{ }^{\mathrm{t}}$-the region of the evacuation system at t time step; $S_{m}^{\mathrm{t}}$-the evacuation region around $\mathrm{m}$-th exits at $\mathrm{t}$ time step . We define $S_{i n}^{\mathrm{t}}=W * W, S_{m}^{\mathrm{t}}=\frac{\pi \cdot\left(R_{x y}^{m}\right)^{2}}{2}, K_{i n}^{t}$ is the density of evacuation system, $K_{m}^{t}$ is the density of the count-area. They reflect the level of crowding about the evacuation system and the count-areas of the m-th exit. If some pedestrians exist in the count-area for exit $\mathrm{m}$.

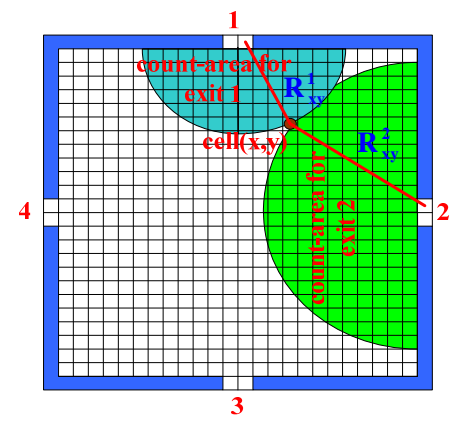

FIGURE II. THE COUNT-AREA FOR EXIT m

\section{RULES}

In pedestrian evacuation simulation model, the time domain can be discretized into a series of finite time steps $t_{1}$, $t_{2} \ldots t_{i}$, a set of intelligent local rules are introduced into our model:

a) The pedestrian can only move one cell and has nine possible target positions to select in each time step.

b) Define transition payoff $\mathrm{P}_{i j}$ as:

$$
\mathrm{P}_{\mathrm{ij}}=\alpha \mathrm{D}_{\mathrm{ij}}+\beta \mathrm{E}_{\mathrm{ij}}+\gamma \mathrm{C}_{\mathrm{ij}}
$$

In every time step, the target cell is chosen by each pedestrian based on the transition payoffs $\mathrm{P}_{i j}$.The pedestrian would choose the cell with the largest value $\mathrm{P}_{M}$ in the matrix of transition payoff as his or her target position. $\mathrm{P}_{M}=\max \left(\mathrm{P}_{i j}\right)$, and $\alpha+\beta+\gamma=1$.

c) A conflict occurs when any two or more pedestrians attempt to move to the same target position. In this situation, each pedestrian enters to the target position with a probability determined by the distance between pedestrian and target cell. One of them will be chosen, the selected pedestrian moves to the corresponding cell and the others stay at the original position. In fact, the closer to the target cell, the greater probability to be selected.

$$
\mathrm{T}_{\mathrm{i}}=\frac{1-\frac{\Delta \mathrm{d}_{\mathrm{i}}}{\sum_{\mathrm{i}=1}^{\mathrm{n}} \Delta \mathrm{d}_{\mathrm{i}}}}{\mathrm{n}-1}
$$

where $T_{\mathrm{i}}$ - the probability of $i$-th pedestrian will be chose; $\Delta \mathrm{d}_{\mathrm{i}}$ - the distance between $i$-th pedestrian and target cell; $n$ - the quantity of pedestrian enter the target cell; 

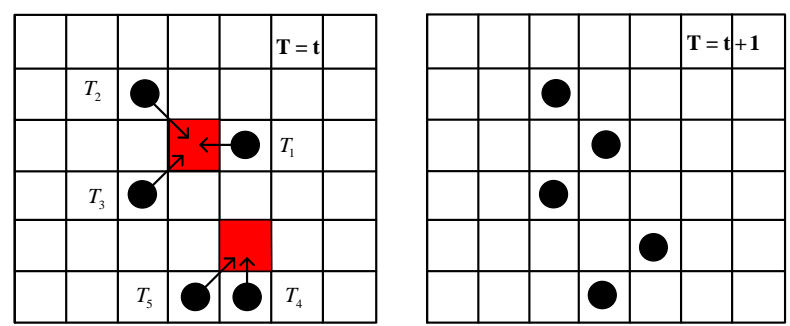

FIGURE III. POSSIBLE MOVEMENT FOR PEDESTRIANS IN OUR CA MODEL WHEN A CONFLICT OCCURS.

d) If two or more neighboring cells have the same transition payoff, one of them will be chosen as the target position through the different probability based on the distance between possible cell and exit. Sometimes pedestrian prefer to move into the cellular which closer to the door as the target position, a great probability of entering the cell which nearer to the exit, however, the distance is from possible cell to the $m$-th exit, the value is equal to $R_{\mathrm{xy}}^{m}$ :

$$
\mathrm{U}_{\mathrm{j}}=\frac{1-\frac{R_{\mathrm{xyj}}^{m}}{\sum_{\mathrm{j}=1}^{\mathrm{n}} R_{\mathrm{xyj}}^{m}}}{\mathrm{n}-1}
$$

where $U_{\mathrm{j}}$-the probability of $j$-th cell will be chose; $R_{\mathrm{xyj}}^{m}-$ the distance between $j$-th cell and $m$-th exit, $(\mathrm{x}, \mathrm{y})$ is the coordinate of $j$-th cell; $n$-the quantity of cells have the same transition payoff.
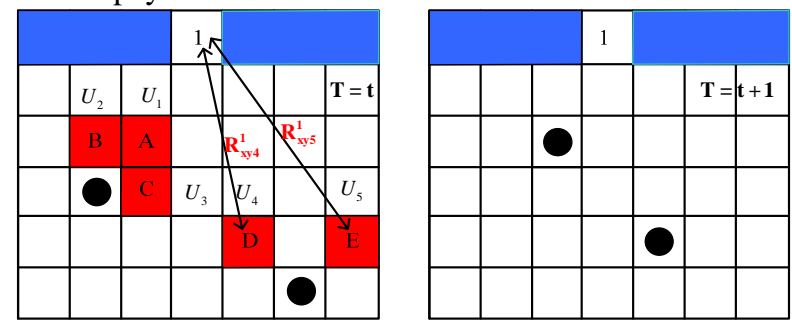

FIGURE IV. POSSIBLE MOVEMENT FOR PEDESTRIANS IN OUR CA MODEL WHEN NEIGHBORING CELLS HAVE THE SAME TRANSITION PAYOFF.

e) Within a time step, if and only if two pedestrians simultaneously choose each other's presently occupied cell as their target position, will the mutual position exchange occur between the two pedestrians. When the position exchange takes place, the action is successfully fulfilled. When the conditions of the pedestrian exchanging position are not met, the pedestrians stay at the original position and will not move to any other cell.

f) When a pedestrian moves into the exit cell, at the next time step the pedestrian will leave the room system. After leaving the room, the pedestrian would not reenter the room system.

g) When all the pedestrians leave the room and there is no pedestrian in the evacuation room, the simulation procedure is terminated. h) Do above until all the pedestrians finish their evacuation.

\section{SimULATION ANALYSIS}

The pedestrians are distributed randomly in the system and there is no pedestrian in the exit cell. The effect of exits layout on the evacuation time is examined through the simulation, in which four exits are set at walls as follow:

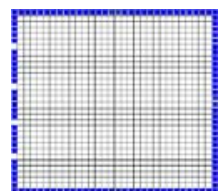

(a) $\mathrm{ID}=1$

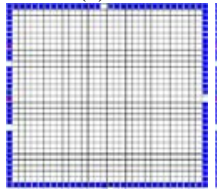

(e) ID=5

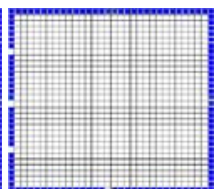

(b) ID=2

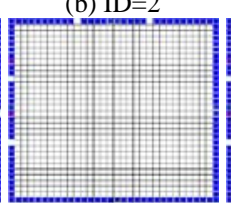

(f) ID $=6$

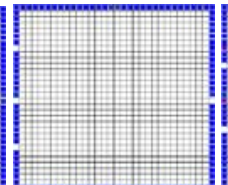

(c) ID=3

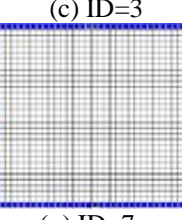

(g) $\mathrm{ID}=7$

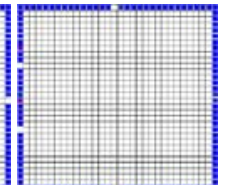

(d) $\mathrm{ID}=4$
FIGURE V. SCHEMATIC ILLUSTRATION OF THE FOUR EXITS ARRANGEMENT

In Figure VI, we plot the evacuation time $\mathrm{T}$ (in time steps units) versus exit width $\mathrm{L}$ while $\mathrm{W}$ is fixed $(\mathrm{W}=50)$. While system size is determinedevacuation time $\mathrm{T}$ decreases nonlinearly (negative exponential distribution) when $\mathrm{L}$ is increased, because the pedestrian is randomly distributed in the system at the beginning of evacuation, after the evacuation started, pedestrian rapidly flocking to the exit and thus form a semi-circular queue. With the increasing of exit width, the capacity as well as the outflow of exits also increasing, so queuing phenomenon has alleviated and evacuation time decreases when exit width is increased. Eventually reaching a saturation state where further increase in exit width does not have major effects on evacuation time, because the utilization rate of exit for pedestrian evacuation getting lower.

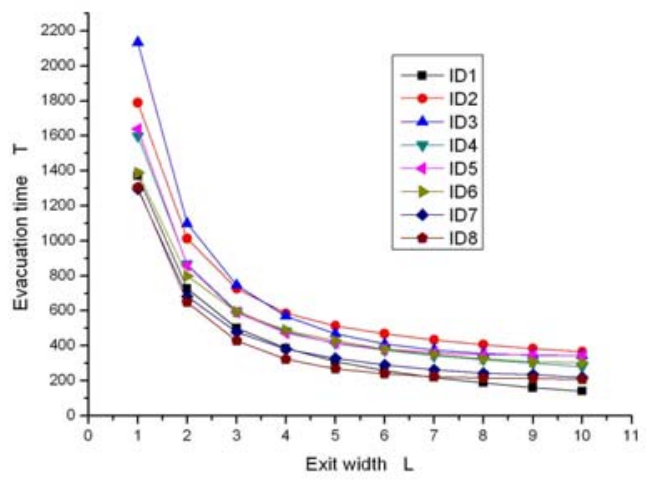

FIGURE VI. EVACUATION TIME VERSUS EXIT WIDTH L 


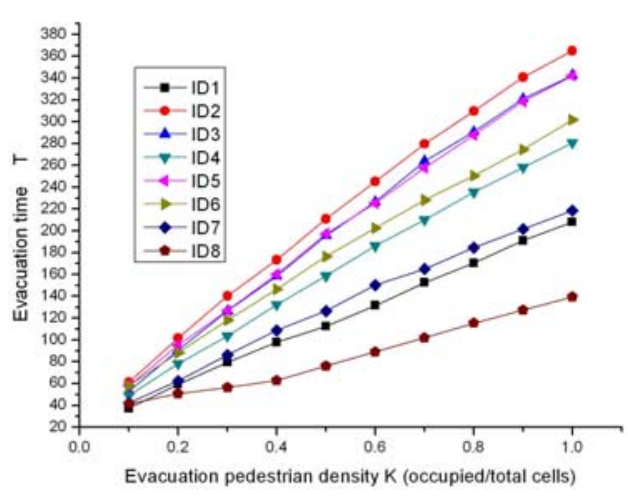

\section{FIGURE VII. EVACUATION TIME T VERSUS EVACUATION PEDESTRIAN DENSITY K}

In Figure VII, we plot the evacuation time $\mathrm{T}$ (in time steps units) versus evacuation pedestrian density $\mathrm{K}$ while $\mathrm{W}$ is fixed $(\mathrm{W}=50)$. While system size is determined, evacuation time $\mathrm{T}$ increases linearly when evacuation pedestrian density $\mathrm{K}$ is increased. Because when pedestrian density is small, nearly all pedestrians can be no conflict to their respective target grid, thus the average speed of all pedestrians much greater and evacuation time is small. However, with the pedestrian density gradually increasing, pedestrian movement become difficult, the average speed of evacuation relatively small, so evacuation time increases under this condition. At the same time, we can see that when system size is determined, evacuation time $\mathrm{T}$ decreases when exit width $L$ is increased. Figure VIII shows the snapshots of evacuation process while $\mathrm{W}=30, \mathrm{~L}=2$, and $\mathrm{K}=0.1$.

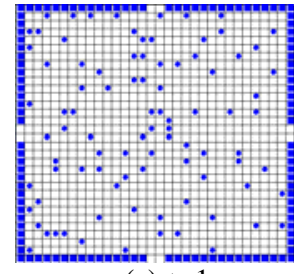

(a) $t=1$

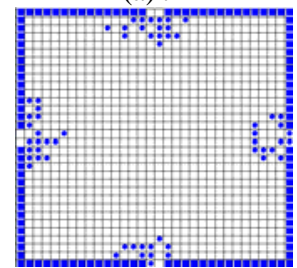

(d) $t=15$

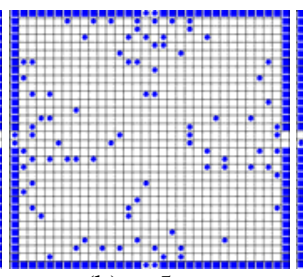

(b) $t=5$



(e) $t=20$

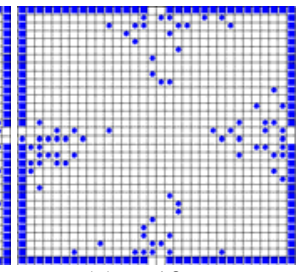

(c) $t=10$

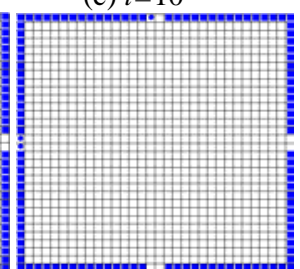

(f) $t=27$
FIGURE VIII. CONFIGURATION OF THE EVACUATING PEDESTRIAN FLOW WITH ID=8, W=30, L=2, AND K=0.1

\section{CONCLUSIONS}

In this paper, simulation of pedestrian evacuation flow from a room with asymmetrical exit layout is presented based on cellular automata (CA). The room without obstacles, a simple scaling law is proposed to model the dependence of evacuation time with the number of persons and exit width, according to two-dimensional cellular automaton Moore neighborhood. The dynamic parameters: Direction-parameter, Empty-parameter and Cognition-parameter are formulated to simplify tactically the decision-making process of pedestrians, considering the effects of pedestrian jam around exits and the width of exits on evacuation path selection in order to reduce evacuation imbalance caused by the asymmetry of exits layout, which can reflect the pedestrian judgment on the surrounding conditions and decide the pedestrian's choice of action. The simulation results of the improved and previous models are compared and analyzed. The relationships of evacuation time versus pedestrian density and exit width for different sizes of systems are studied. Simulation results show that evacuation time $\mathrm{T}$ increases nonlinearly (exponential distribution) when system size is increased, there is a linear relationship between evacuation time and pedestrian density, however, there is a negative exponential relationship between evacuation time and exit width. The simulation results correspond with the actual, it is instructional significant for pedestrian evacuation. In our model, we consider the effects of the crowd distribution in evacuation process, to provide the occupants safety in enclosed environments, avoiding and/or reducing the number injuries.

\section{ACKNOWLEDGMENT}

This paper is financially supported by the National Natural Science Foundation of China (Nos. 11422221).

\section{REFERENCES}

[1] C. Burstedde, K. Klauck, A. Schadschneider, J. Zittartz, Simulation of pedestrian dynamics using a two-dimensional cellular automaton, Physica A 295 (2001) 507-525.

[2] D. Helbing, P. Molnar, Social force model for pedestrian dynamics, Phys. Rev.E 51 (5) (1995) 4282-4286.

[3] M. Muramatsu, T. Irie, T. Nagatani, Jamming transition in pedestrian counter flow, Physica A 267 (1999) 487-498.

[4] D. Helbing, I. Farkas, T. Vicsek, Simulating dynamical features of escape panic, Nature 407 (2000) 487-490.

[5] C.M. Henein, T.White, Macroscopic e®ects of microscopic forces between agents in crowd models, Physica A 373 (2007) 694-712.

[6] W. Song, X. Xu, B.Wang, S. Ni, Simulation of evacuation processes using a multi-grid model for pedestrian dynamics, Physica A 363 (2006) 492-500.

[7] S. Liu, L. Yang, T. Fang, J. Li, Evacuation from a classroom considering the occupant density around exits, Physica A 388 (2009) 1921-1928.

[8] W. Yuan, K. Tan, An evacuation model using cellular automata, Physica A 384(2007) 549-566.

[9] H. Yue, C. Shao, X. Chen, H. Hao, Simulation of bi-directional pedestrian flow based on cellular automata model, Acta Phys. Sinica 57 (2008) 6901-6908.

[10] Zhu Nuo, Jia Bin, Shao Chun-fu, Yue Hao. Simulation of pedestrian evacuation based on an improved dynamic parameter model, Chinese Physics B.21(5) ( 2012). 050501.

[11] Zhu Nuo, Jia Bin, Shao Chun-fu. Pedestrian evacuation based on a dynamic parameters model. Applied Mechanics and Materials, 9798(2011) 956-959

[12] Nuo Zhu. Pedestrian evacuation with obstacles based on dynamic parameters model. Computer Science and Engineering, 40-44 (2017). 